\title{
Unleashing The Potential Of Multiple-Targeted Therapies In Hepatobiliary Cancers: Two Cases, Cocktail Therapy With Nine Molecular Targeted Agents And Long-Lasting Survival
}

This article was published in the following Dove Press journal:

OncoTargets and Therapy

Jianzhen Lin

Xiaobo Yang

Yuan Xie

Haitao Zhao

Department of Liver Surgery, Peking Union Medical College Hospital, Chinese Academy of Medical Sciences and Peking Union Medical College (CAMS \& PUMC), Beijing, People's Republic of China
Correspondence: Haitao Zhao Department of Liver Surgery, Peking Union Medical College Hospital, Chinese Academy of Medical Sciences and Peking Union Medical College (CAMS \& PUMC), \#I Shuaifuyuan, Wangfujing, Beijing 100730, People's Republic of China Tel +86-10-69|56042

Fax +86-10-69156043

Email zhaoht@pumch.cn

\begin{abstract}
The mortality of hepatobiliary cancer still stays high around the world, with disappointing treatment status at advanced stage. Genomic analysis has identified the global spectrum of alterations in liver cancer, but the most common mutations are not actionable, meanwhile lacking potent molecular-targeted drugs for specific oncogenic drivers. Multipletargeted drugs which mainly inhibited tumors' angiogenesis, such as sorafenib and lenvatinib, improved survival time for patients with advanced hepatocellular carcinoma, although the median overall survival remains $1-1.5$ years. It is still ambiguous for the precise biomarkers of multiple-targeted drugs utilized in hepatobiliary cancer. Herein, we reported two observational patients who were sequentially treated by various targeted drugs and obtained lasting overall survival time. Both the two patients weekly switched targeted drugs according to the changes of tumor markers. These two cases would get us thinking: what is the underlying mechanism of molecular-targeted drugs in hepatobiliary tumors; and whether a "drugs screening model" during real-time treatment could be achieved through the assistance from sensitive and specific tumor markers.
\end{abstract}

Keywords: hepatobiliary cancer, molecular targeted drug, precision medicine

Effective and proven systematic adjuvant chemotherapy regimens for advanced hepatobiliary malignancies remain of limited clinical utility and typically fail to show effective and enduring responses. To this end, advances in cancer genomics and targeted use of molecular-directed agents have brought about new therapeutic options for patients with hepatobiliary tumors. ${ }^{1,2}$ Previous studies investigating genomic characteristics of cholangiocarcinoma have revealed that actionable alterations mainly included IDH1/2, FGFR fusion and sporadic pathogenic mutations in BRCA1/2 and mismatch repair genes. ${ }^{2}$ Thus, investigation of numerous pathwaytargeted or oncogenic signaling-targeted therapies holds promise for patients with hepatobiliary tumors. We have launched a clinical trial termed "Precise Treatments in Hepatobiliary Cancers (PTHBC, NCT02715089)," in March 2016. Our trial was based on genetic analyses and precise diagnosis of tumors to explore precise treatments in hepatobiliary cancer. Important goals were to evaluate drug safety, progression-free clinical course and finally overall survival.

Here, we retrospectively reported and discussed two illustrative patients, who were diagnosed and observed during this study time course. Each of these two 
end-stage patients received multiple targeted therapeutic agents (up to nine different drugs, termed "targeted cocktail therapy").

\section{Case I}

A 73-year-old male patient was diagnosed with advanced cholangiocarcinoma with extensive and multi lesions in the liver (greatest diameter: $10.7 \mathrm{~cm}$ ) without liver cirrhosis. He has a history of hepatitis type B infection for 21 years but is negative for schistosomiasis infection. Biopsies confirmed moderately differentiated cholangiocarcinoma positive for CD34 (1+), CK7 (3+) and CK19 (3K). The patient underwent percutaneous transhepatic cholangial drainage for relief of obstructive jaundice. In the absence of effective anti-tumor therapy, however, the tumor progressed after 2 months of initial diagnosis. The cholangiocarcinoma tissue biopsies were sent to proceed molecular expression detection.

\section{Molecular Pathology Results}

Immunohistochemistry in situ indicated high expression of EGFR (2+), VEGFR (3+), C-erbB2 (3+), with $30 \%$ positive for Ki-67.

DNA extraction was inadequate for oncogene sequencing so the tumor genetic profiling was uncertain.

\section{Treatment And Clinical Course}

The hospital multidisciplinary tumor board recommended standard systematic chemotherapy (gemcitabine plus oxaliplatin). The patient refused chemotherapy and then administered the mixed cocktail targeted therapy by himself, with nine molecular-specific agents (Figure 1). The exact therapeutic process and sequential tumor markers (TM, CA19-9 and CA125) are as summarized in Figure 1 and reviewed as follow:

The patient chose afatinib ( $40 \mathrm{mg}$ once daily) as the firstline treatment for the high expression of EGFR and C-erbB2. The 39-day treatment achieved stability in clinical disease (radiological assessment referred to RECIST v1.1 ${ }^{3}$ ) but as TM remained elevated, he was then treated with cabozantinib (40 mg twice daily), a low molecular inhibitor of the receptor tyrosine kinases (RTK; e.g. c-Met and VEGFR2) given high expression of the latter receptor in the tumor.

After the 10-day treatment, the TM did not sharply decline so he turned to dacomitinib (targeting both EGFR and C-erbB2). This course of dacomitinib (30 mg once daily for 13 days) did not lower TM. However, radiological imaging indicated decreases in tumor size. At that time, therapy was changed to sunitinib $(25 \mathrm{mg}$ once daily), a small molecule RTK inhibitor, for 12 days, with dramatical declines in TM. Patient then restarted

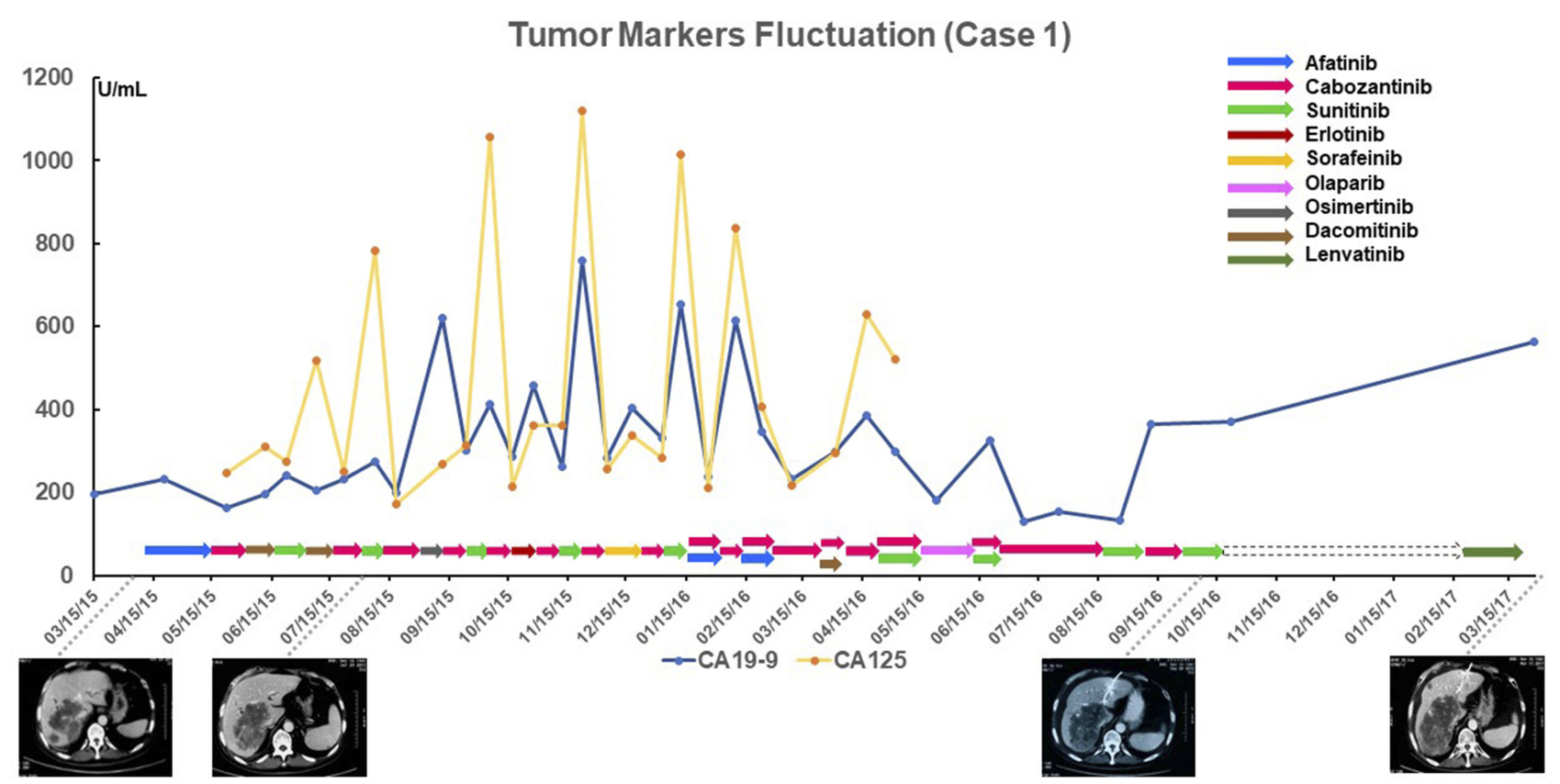

Figure I Overview of the therapeutic regimens and tumor marker fluctuations in patient case I, who used up to nine different targeted drugs over a 2 years period. The arrows under the line chart indicate the therapeutic regimen for each time (dash arrow means period of targeted therapy pause). The central curves indicate fluctuations of tumor markers. 
dacomitinib for 12 days but the CA125 then increased rapidly.

He then formed anti-VEGFR modalities with cabozantinib and/or sunitinib to control tumor progression. The patient used cabozantinib or sunitinib alternatively every fortnight, interspersing this regimen with anti-EGFR targeted therapy, with the use of osimertinib $(80 \mathrm{mg}$ once daily) or erlotinib (100 mg once daily), which was then monitored by TM. Intriguingly, the tumor lesions were stable without evidence for distant metastasis for 17 months. Overall, the adverse events (AEs) induced by the molecular-targeted drugs were tolerable, and no severe AEs were reported by the patient, possibly due to the low dosage and short enduring time of each drug. However, after long-term medication and recurrence of obstructive jaundice, he suspended targeted treatment for 3 months and turned to supportive care.

Imaging assessment confirmed progressive disease after 3-month time-out from targeted anti-tumor therapy. He restarted alternative targeted treatment with lenvatinib and cabozantinib, but the cancer still progressed. Finally, he died of cancer progression and survived for 31 months since the first time he received targeted therapy.

\section{Case 2}

A 57-year-old male patient underwent right hepatectomy for multi-focal liver malignancy (main lesion was $2.3 \mathrm{~cm}$ ). He had had chronic hepatitis virus $\mathrm{B}$ infection for 20 years and liver cirrhosis for 10 years. The pathology revealed moderately differentiated hepato-cholangiocarcinoma. He received TACE as postoperative adjuvant therapy on one occasion. At 3 months after surgery, tumor recurred and PET-CT scan indicated that intrahepatic tumors invaded the hilar and right hepatic lobe. There was main portal vein invasion, as well as multiple intra-abdominal metastases. The cancer progressed after adjuvant radiotherapy and he required ERCP and stent placement. His tumor tissue was sent to tumor profiling laboratory.

\section{Molecular Pathology Results}

Tumor cells were estimated to constitute around $80 \%$ of the total cells in a sample from the previous surgical resection. Immunohistochemistry in situ indicated no expression of PD-1, PD-L1 and VEGFR. Mild positive expression of EGFR (1+), C-erbB2 (1+, 30\%) was noted.

Cancer genomic sequencing revealed pathogenic somatic mutations in ATM (c.A1561T; p.R521*) and JAK3 (c.C3239G; p.P1080R).

\section{Treatment And Clinical Course}

The PUMCH multidisciplinary tumor board recommended the patient undergo treatment with standard first-line chemotherapy based on gemcitabine for cholangiocarcinoma, alternatively to receive olaparib based on the presence of a pathogenic serine-threonine kinase ATM mutation. He refused the standard form of chemotherapy.

He first received afatinib, because of positive expression of EGFR and C-erbB2 in tumor. One-month afatinib (40 mg once daily) decreased CA19-9 (from 121 to 26.1). Because of oral mucosa ulcerations, he changed to another 10-day cabozantinib (80 mg twice daily), with CA19-9 falling from 26.1 to 19.

However, imaging assessment indicated progressive disease after 1.5 months of targeted therapy. He then employed olaparib (400 mg twice daily) treatment. Onemonth olaparib achieved stable disease (SD) in primary lesion, but a new lesion appeared in right adrenal gland and the CA19-9 became elevated to 42.1. Then, he chose to be alternatively treated by cabozantinib ( $80 \mathrm{mg}$ twice daily) and axitinib (10 mg twice daily) every month. The 3-month post-therapy assessment suggested overall SD with shrinkage of lesions (Figure 2A and B), while at 6.5-month post-therapy, our evaluation confirmed cancer progression with emerging metastases and increase in size of the primary lesions. He was then changed to lenvatinib (12 mg once daily) as salvage anti-cancer treatment, but the one-month single targeted therapy was ineffective. Ultimately, he received immunotherapy with PD-1 inhibitor (pembrolizumab, $200 \mathrm{mg}$ per 3 weeks) for 3 months, and there was no objective evidence of response. He died from cancer cachexia and uncontrolled biliary infection, albeit he survived for 20 months on targeted therapy.

\section{Revelation}

The characteristics of precision medicine provide MTDs with the advantages of better tolerability with less adverse events. However, precision may often also mean narrow range of efficacy. Cancers, particularly hepatobiliary tumors, possess complicated transduction signaling pathways, exhibit global complementary networks in oncogenic process and show much heterogeneity during clonal evolution. ${ }^{4}$ Thus, multiple targeted approaches could likely exhibit better therapeutic efficacy in hepatobiliary tumors.

Here, we presented illustrative end-stage patients treated with targeted cocktail therapy, who appeared to have obtained prolonged survival time with good tolerability. We communicate these results to show how drug selection 
A
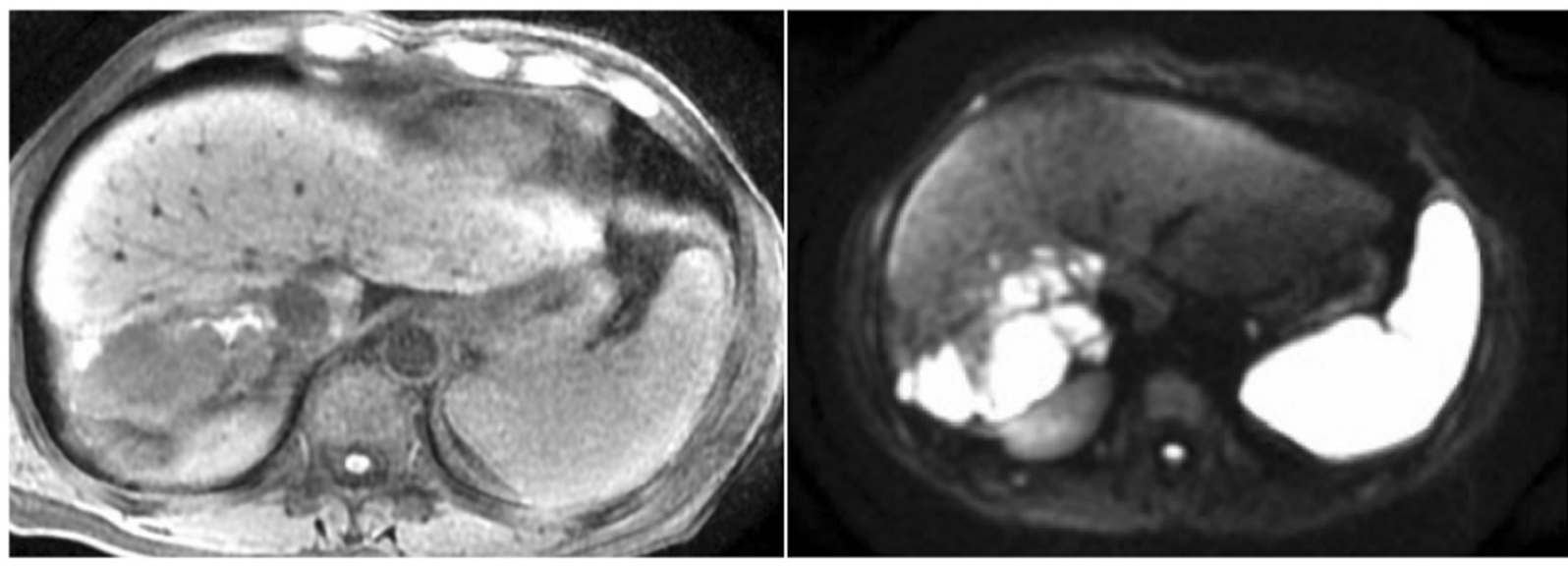

B

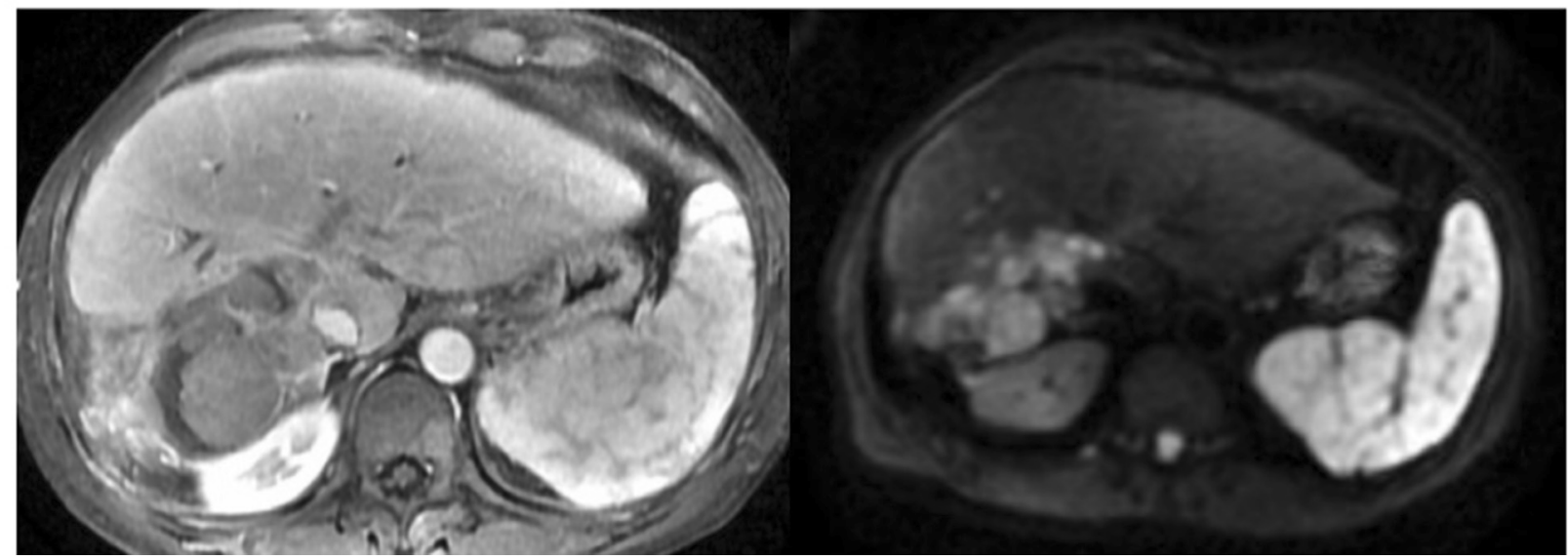

Figure 2 Radiological evidence for the shrank target lesions from the patient in case 2, who received alternative cabozantinib and axitinib for 3 months $((\mathbf{A})$ prior the treatment; (B) post treatment).

may proceed in the relative absence of clear guidelines and lack of direction as to specific molecular targets in hepatobiliary tumors.

Our questions and perspectives arising from these 2 cases are as follows:

1. Is it reasonable to independently guide targeted therapy based merely on cancer-related mutations? The unpredictable outcomes raise questions as to the definition of precise guiding targets for targeted therapy. ${ }^{5}$

2. Is imperative to monitor dynamically during targeted therapy by utilizing sensitive and noninvasive tumor biomarkers, which could timely reflect cancer response with ongoing treatment? Traditional tumor markers might be highlighted to direct clinical management. Sensitive tumor markers could also narrow the therapeutic window period between initiation and efficacy evaluation.

3. Might future standard clinical practice require rapid switch over of next-line treatment options, when progressive disease occurs? Clinical trials have shown that regorafenib can still bring survival benefits for progressive disease, even after sorafenib failure. This is observed despite two of these MTDs target VEGFR, along with other tyrosine kinase receptors. It remains unclear as to whether and how to alternate different and related MTDs during treatment for stable and progressive disease.

4. Is this therapeutic mode suitable for clinical practice in the real-world? From the perspective of costbenefit, most patients do not have the economic strength to undertake long-term treatment with multiple targeted drugs. 


\section{Conclusion}

Overall, our comprehension of the relative contributions of factors including actual validated targets and defining the essential mechanisms of MTDs responses remain major challenges in precision oncology. We still have a long journey to achieve biomarker-guided targeted therapy in hepatobiliary tumors.

\section{Abbreviations}

ATM, ataxia telangiectasia; CA, cancer antigen; EGFR, epidermal growth factor receptor; ERCP, endoscopic retrograde cholangio-pancreatography; $\mathrm{H}-\mathrm{ChC}$, hepatocholangiocellular carcinoma; JAK, janus kinases; MTDs, molecular targeted drugs; RTK, receptor tyrosine kinase; TACE, transcatheter arterial chemoembolization; TM, tumor markers; SD, stable disease; VEGFR, vascular endothelial growth factor receptor.

\section{Ethics Approval And Consent To Participate}

All patients participated in this observational trial provided written informed consent for medical treatment and collection of tumor biopsies and for comprehensive molecular profiling of tumor and germline samples. All patients were enrolled in institutional protocols approved by the Ethics Review Committee of Peking Union Medical College Hospital (Beijing, China) (reference no. PM-1503).

\section{Data Sharing Statement}

The datasets used and/or analyzed during the current study were available from the corresponding authors on reasonable request.

\section{Consent For Publication}

The consent forms were signed by every participant and will be provided upon request.

\section{Acknowledgments}

The authors thank the patients who volunteered to participate in this study and the staff members at the study sites who cared for these patients; the members of the data and safety monitoring committee; representatives of the sponsors who were involved in the data collection and analyses; and technology support. The authors sincerely thank Dr. Simon C. Robson for his help in revising the manuscript.

\section{Author Contributions}

All authors contributed to data analysis, drafting or revising the article, gave final approval of the version to be published, and agree to be accountable for all aspects of the work.

\section{Funding}

This work is supported by the International Science and Technology Cooperation Projects (2016YFE0107100 and 2015DFA30650), the CAMS Innovation Fund for Medical Science (CIFMS) (2017-I2M-4-003), the Beijing Natural Science Foundation (L172055), the National Ten-thousand Talent Program, Beijing Science and Technology Cooperation Special Award Subsidy Project and CAMS Initiative for Innovative Medicine (CAMS-2018-I2M-3-001).

\section{Disclosure}

The authors declare no conflicts of interest.

\section{References}

1. Llovet JM, Montal R, Sia D, Finn RS. Molecular therapies and precision medicine for hepatocellular carcinoma. Nat Rev Clin Oncol. 2018. doi:10.1038/s41571-018-0073-4

2. Valle JW, Lamarca A, Goyal L, Barriuso J, Zhu AX. New horizons for precision medicine in biliary tract cancers. Cancer Discov. 2017;7:943-962. doi:10.1158/2159-8290.CD-17-0245

3. Schwartz LH, Seymour L, Litiere S, et al. RECIST 1.1 - standardisation and disease-specific adaptations: perspectives from the RECIST working group. Eur J Cancer. 2016;62:138-145. doi:10.1016/j.ejca.2016.03.082

4. McGranahan N, Swanton C. Clonal heterogeneity and tumor evolution: past, present, and the future. Cell. 2017;168:613-628. doi:10.10 16/j.cell.2017.01.018

5. Dagogo-Jack I, Shaw AT. Tumour heterogeneity and resistance to cancer therapies. Nat Rev Clin Oncol. 2018;15:81-94. doi:10.1038/ nrclinonc.2017.166

\section{Dovepress}

\section{Publish your work in this journal}

OncoTargets and Therapy is an international, peer-reviewed, open access journal focusing on the pathological basis of all cancers, potential targets for therapy and treatment protocols employed to improve the management of cancer patients. The journal also focuses on the impact of management programs and new therapeutic

agents and protocols on patient perspectives such as quality of life, adherence and satisfaction. The manuscript management system is completely online and includes a very quick and fair peer-review system, which is all easy to use. Visit http://www.dovepress.com/ testimonials.php to read real quotes from published authors. 